University of New Orleans

ScholarWorks@UNO

8-1983

\title{
Simple and direct determination of complex refractive index and thickness of unsupported or embedded thin films by combined reflection and transmission ellipsometry at $45^{\circ}$ angle of incidence
}

R. M.A. Azzam

University of New Orleans, razzam@uno.edu

Follow this and additional works at: https://scholarworks.uno.edu/ee_facpubs

Part of the Electrical and Electronics Commons, and the Physics Commons

\section{Recommended Citation}

R. M. A. Azzam, "Simple and direct determination of complex refractive index and thickness of unsupported or embedded thin films by combined reflection and transmission ellipsometry at $45^{\circ}$ angle of incidence," J. Opt. Soc. Am. 73, 1080-1082 (1983)

This Article is brought to you for free and open access by the Department of Electrical Engineering at ScholarWorks@UNO. It has been accepted for inclusion in Electrical Engineering Faculty Publications by an authorized administrator of ScholarWorks@UNO. For more information, please contact scholarworks@uno.edu. 


\title{
Simple and direct determination of complex refractive index and thickness of unsupported or embedded thin films by combined reflection and transmission ellipsometry at $45^{\circ}$ angle of incidence
}

\author{
R. M. A. Azzam \\ Department of Electrical Engineering, University of New Orleans, Lakefront, New Orleans, Louisiana 70148
}

Received February 4, 1983

\begin{abstract}
Measurements of the polarization states (represented by complex numbers $\chi_{r}$ and $\chi_{t}$, respectively) of light reflected and transmitted by an unsupported or embedded thin film, for totally polarized light (with nonzero $p$ and $s$ components) incident at $45^{\circ}$, permit simple, direct, and explicit determination of the film's complex refractive index $N_{1}$ independently of film thickness or input polarization. If $\alpha=\chi_{r} / \chi_{t}$, we find that $\alpha=r_{s}+r_{s}^{-1}$, where $r_{s}$ is Fresnel's complex reflection coefficient of the ambient-film interface for the $s$ polarization at $45^{\circ}$ incidence. From $\alpha, r_{s}$ is determined, and from $r_{s}$ we get $N_{1}=N_{0}\left(1+r_{s}^{2}\right)^{1 / 2} /\left(1+r_{s}\right)$, where $N_{0}$ is the refractive index of the transparent medium surrounding the film. Knowledge of the incident polarization $\chi_{i}$ allows the film thickness to be determined, also explicitly, by using either of the ratios $\chi_{i} / \chi_{r}$ or $\chi_{i} / \chi_{t}$.
\end{abstract}

\section{INTRODUCTION}

The inverse problem in ellipsometry, ${ }^{1}$ in which ratios of complex reflection or transmission coefficients determined from polarization measurements are used to find unknown optical properties (including film thicknesses) of a stratified structure, is of long-standing interest. Even for the simple and often-adopted idealized three-phase (ambient-filmsubstrate) model (with homogeneous isotropic phases separated by sharp parallel-plane boundaries), the inverse problem defies analytical inversion in all but one known case. This is when the optical properties of all media are known and only the film's thickness is to be determined, ${ }^{2}$ a case that is of limited practical interest.

In this Letter we show that combined reflection and transmission ellipsometry ${ }^{3}$ at a $45^{\circ}$ angle of incidence, on an absorbing thin film bounded by a transparent medium of known refractive index (i.e., a two-phase system that consists of a thin-film phase and a surrounding bulk phase), permits simple and explicit determination of the complex refractive index and thickness of the film.

\section{METHOD}

Figure 1 shows the reflection and transmission of collimated monochromatic totally polarized light by a plane-parallel thin film of thickness $d$, medium 1, surrounded by a transparent medium 0 . Both media are assumed to be homogeneous and optically isotropic. $\phi$ is the angle of incidence $\left(45^{\circ}\right)$, and $p$ and $s$ indicate the linear polarizations parallel and perpendicular to the plane of incidence, respectively.

If we approximate the incident beam by a plane wave, the complex amplitude-reflection and -transmission coefficients for the $\nu$ polarization $(\nu=p, s)$ are given by ${ }^{4}$

$$
R_{\nu}=\left(r_{01 \nu}+r_{10 \nu} X\right) /\left(1+r_{01 \nu} r_{10 \nu} X\right) \text {, }
$$

$$
T_{\nu}=t_{01 \nu} t_{10 \nu} X^{1 / 2} /\left(1+r_{01 \nu} r_{10 \nu} X\right),
$$

where

$$
\begin{gathered}
X=\exp \left(-j 2 \pi d / D_{\phi}\right), \\
D_{\phi}=\frac{\lambda}{2}\left(N_{1}{ }^{2}-N_{0}^{2} \sin ^{2} \phi\right)^{-1 / 2} .
\end{gathered}
$$

In Eqs. (1) and (2), $r_{m n \nu}$ and $t_{m n \nu}$ are Fresnel's reflection and transmission coefficients, respectively, of the $m n$ interface for the $\nu$ polarization. In Eq. (4), $\lambda$ is the free-space wavelength of light, and $N_{0}$ and $N_{1}$ are the real and complex refractive indices of the ambient and the film, respectively.

Fresnel's coefficients at the 01 and 10 interfaces are interrelated by ${ }^{5}$

$$
\begin{gathered}
r_{01 \nu}=-r_{10 \nu}=r_{\nu}, \\
t_{01 \nu} t_{10 \nu}=\left(1-r_{\nu}{ }^{2}\right) .
\end{gathered}
$$

Substitution of Eqs. (5) and (6) into Eqs. (1) and (2) gives

$$
\begin{gathered}
R_{\nu}=r_{\nu}(1-X) /\left(1-r_{\nu}{ }^{2} X\right), \\
t_{\nu}=\left(1-r_{\nu}{ }^{2}\right) X^{1 / 2} /\left(1-r_{\nu}{ }^{2} X\right) .
\end{gathered}
$$

From Eqs. (7) and (8), the ratios of $p$ and $s$ reflection and transmission coefficients,

$$
\rho_{r}=R_{p} / R_{s}, \quad \rho_{t}=T_{p} / T_{s},
$$

are given by

$$
\begin{gathered}
\rho_{r}=\left(r_{p} / r_{s}\right)\left(1-r_{s}^{2} X\right) /\left(1-r_{p}^{2} X\right), \\
\rho_{t}=\left[\left(1-r_{p}^{2}\right) /\left(1-r_{s}^{2}\right)\right]\left[\left(1-r_{s}^{2} X\right) /\left(1-r_{p}^{2} X\right)\right] .
\end{gathered}
$$

In ellipsometry, measurements of the polarization states of the incident $(i)$, reflected $(r)$, and transmitted $(t)$ waves determine $\rho_{r}$ and $\rho_{t}$. Thus, if polarization is represented by the complex number ${ }^{6}$ 


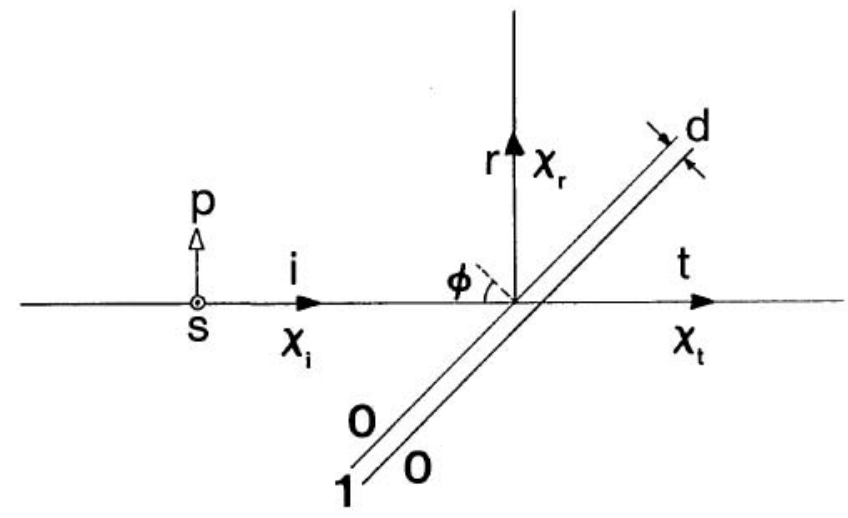

Fig. 1. A light beam (i) incident at $\phi=45^{\circ}$ is split into reflected $(r)$ and transmitted $(t)$ components by an unsupported or embedded thin film of thickness $d$, medium 1 , which is in contact on both sides with a transparent medium $0 . p$ and $s$ indicate linear polarizations parallel and perpendicular to the plane of incidence. $\chi_{i}, \chi_{r}$, and $\chi_{t}$ represent the polarization states of the incident, reflected, and transmitted beams, respectively.

$$
\chi_{k}=E_{k s} / E_{k p}, \quad k=i, r, t,
$$

where $E_{k s}$ and $E_{k p}$ are the $s$ and $p$ phasor components of the electric vector of the $k$ th wave, we find that

$$
\rho_{r}=\chi_{i} / \chi_{r}, \quad \rho_{t}=\chi_{i} / \chi_{t} .
$$

In general, Eqs. (10) and (11) overdetermine the film properties (its complex refractive index $N_{1}$ and thickness $d$ ) in terms of $\rho_{r}$ and $\rho_{t}$. The inversion problem is greatly simplified if we form the ratio

$$
\alpha=\rho_{t} / \rho_{r}
$$

and substitute from Eqs. (10) and (11) to obtain

$$
\alpha=\left(r_{s} / r_{p}\right)\left(r_{p}^{2}-1\right) /\left(r_{s}^{2}-1\right) .
$$

Significantly, $\alpha$ is independent of $X$ and hence of film thickness. Furthermore, if Eqs. (13) are substituted into Eq. (14), we get

$$
\alpha=\chi_{r} / \chi_{t}
$$

which shows that $\alpha$ is determined only by the reflected and transmitted polarization states, independently of the incident polarization. (The latter must, of course, have nonzero $p$ and $s$ components.)

For a known ambient refractive index $N_{0}$, Eq. (15) has the complex refractive index of the film, $N_{1}$, as its only unknown. The dependence of $\alpha$ on $N_{1}$ is implicit in the Fresnel coefficients $r_{p}$ and $r_{s}$ of the ambient-film interface.

To solve Eq. (15) for $N_{1}$, further simplification is achieved by choosing the angle of incidence $\phi=45^{\circ}$. At this angle, Abelès ${ }^{7}$ has shown that

$$
r_{p}=r_{s}{ }^{2} .
$$

Equation (17) reduces Eq. (15) to

$$
\alpha=r_{s}+\frac{1}{r_{s}},
$$

from which

$$
r_{s}=\frac{1}{2}\left[\alpha \pm\left(\alpha^{2}-4\right)^{1 / 2}\right]
$$

Of the two roots, only that for which $\left|r_{s}\right|<1$ has physical significance.

It is interesting that the complex reflection coefficient for the $s$ polarization, $r_{s}$, of the ambient-film interface can be determined from the measured polarization states of the reflected and transmitted waves. From $r_{s}$, we finally obtain 8

$$
N_{1}=N_{0}\left(1+r_{s}^{2}\right)^{1 / 2} /\left(1+r_{s}\right) \text {. }
$$

To determine film thickness, the incident polarization $\chi_{i}$ must be known (e.g., $\chi_{i}=1$, when a linear polarizer is placed in the incident beam with its transmission axis at $45^{\circ}$ azimuth from the plane of incidence), so that $\rho_{r}$ or $\rho_{t}$ is determined by one of Eqs. (13). Subsequently, Eq. (10) or (11) is solved for $X$. With $r_{p}=r_{s}^{2}$ at $\phi=45^{\circ}$, Eq. (10) gives

$$
X=\left(\rho_{r}-r_{s}\right) / r_{s}{ }^{3}\left(\rho_{r} r_{s}-1\right) .
$$

From $\rho_{r}$ and $r_{s}$, complex $X$ is calculated by using Eq. (21). Next, $d$ is determined from $X$ by Eq. (3):

$$
d=\left(j D_{\phi} / 2 \pi\right) \ln X,
$$

where $D_{\phi}$ is given by Eq. (4). For an absorbing film, Eq. (22) must provide a unique real value for $d$. For a transparent film, $d$ is determined up to an integral multiple of the filmthickness period $D_{\phi}$. Any small imaginary part of $d$ must be considered an error caused by an error either of polarization measurements or of the model used to describe the film (e.g., the assumption of optical isotropy or homogeneity). An additional check is provided by determining $d$ from $\rho_{t}$ by solving Eq. (11) for $X$ and using Eq. (22). Of course, the result must agree, to within an acceptable limit of error, with the determination of $d$ from $\rho_{r}$.

\section{SUMMARY}

Combined reflection and transmission ellipsometry at a $45^{\circ}$ angle of incidence permits simple, explicit, and direct determination of the complex refractive index and thickness of an absorbing thin film that is bounded on both sides by transparent media of the same known refractive index. Such films may be unsupported ${ }^{9}$ (such as ultrathin metal foils ${ }^{10}$ ), immersed in a liquid (e.g., membranes), embedded in a surrounding solid phase, or deposited on a solid substrate and immersed in an index-matched liquid. Significantly, the film's complex refractive index is determined from the measured polarization states of the reflected and transmitted waves, independently of film thickness or incident polarization, and, from knowledge of the incident polarization (which must differ from the $p$ or $s$ state), the film thickness can be found.

In conclusion, we have found a simple solution to the inversion problem of reflection and transmission ellipsometry on an absorbing thin film surrounded by a transparent bulk phase.

\section{ACKNOWLEDGMENT}

I am pleased to acknowledge support by the National Science Foundation under grant DMR-8018417.

Note added in proof: Measurements on an ultrathin gold foil, kindly provided by Gary Reeves of Los Alamos National Laboratory, confirmed the validity of this technique. These results were presented at the Paris Ellipsometry Conference, June 7-10, 1983. 


\section{REFERENCES}

1. R. M. A. Azzam and N. M. Bashara, Ellipsometry and Polarized Light (North-Holland, Amsterdam, 1977), Sec. 4.5.

2. F. L. McCrackin and J. P. Colson, "Computational techniques for the use of the exact Drude equations in reflection problems," in Ellipsometry in the Measurement of Surfaces and Thin Films, E. Passaglia, R. R. Stromberg, and J. Kruger, eds., Misc. Pub. No. 256 (National Bureau of Standards, Washington, D.C., 1964), pp. 61-82.

3. R. M. A. Azzam, M. Elshazly-Zaghloul, and N. M. Bashara, "Combined reflection and transmission thin-film ellipsometry: a unified linear analysis," Appl. Opt. 14, 1652-1663 (1975); I. Ohlídal and F. Lukeš, "Optical analysis of thin gold films by combined reflection and transmission ellipsometry," Thin Solid Films 85, 181-190 (1981).

4. See, for example, Ref. 1, Sec. 4.3 .

5. Ref. 1, p. 283.

6. Ref. 1, Sec. 1.7. $\chi=(\tan \theta+j \tan \epsilon) /(1-j \tan \theta \tan \epsilon)$, where $\theta$ and $\epsilon$ are the major-axis azimuth (measured from the $p$ direction) and $\epsilon$ is the ellipticity angle of the polarization ellipse.

7. F. Abelès, "Un théorème relatif à la réflexion metallique," C. R. Acad. Sci. 230, 1942-1943 (1950).

8. See, for example, R. M. A. Azzam, "Direct relation between Fresnel's interface reflection coefficients for the parallel and perpendicular polarizations," J. Opt. Soc. Am. 69, 1007-1016 (1979), Eq. (19).

9. Films of this type have been extensively characterized by transmission interferometry. See J. Casset, "Transmission optique de lames minces sans support de métaux nobles dans le visible et le proche ultraviolet," Thin Solid Films 18, 99-103 (1973); "Méthode nouvelle de detérmination simultanée de l'épaisseur et de l'indice complexe de lames absorbantes sans support," Thin Solid Films 41, 315-328 (1977); "Optical constants of absorbing materials by transmission interferometry on medium-thickness films," J. Opt. Soc. Am. 69, 725-730 (1979).

10. Ultrathin sheets of gold foil of thickness less than $25 \mathrm{~nm}$ have recently been produced at Los Alamos National Laboratory. See Indust. Res. Develop. 25(3), 43 (1983). 